\title{
A Multi-Sensor Tight Fusion Method Designed for Vehicle Navigation
}

\author{
Qifeng Lai ${ }^{1,2}$, Hong Yuan ${ }^{1, *}$, Dongyan Wei ${ }^{1}$, Ningbo Wang ${ }^{1}$, Zishen $\mathrm{Li}^{1}{ }^{1}$ (I) and Xinchun Ji ${ }^{1}$ \\ 1 Aerospace Information Research Institute, Chinese Academy of Science, Beijing 100864, China; \\ laiqf@aircas.ac.cn (Q.L.); weidy@aircas.ac.cn (D.W.); wangningbo@aoe.ac.cn (N.W.); \\ lizishen@aoe.ac.cn (Z.L.); jixc@aircas.ac.cn (X.J.) \\ 2 School of Electronic, Electrical and Communication Engineering, University of Chinese Academy of Sciences, \\ Beijing 100864, China \\ * Correspondence: yuanhong@aircas.ac.cn
}

Received: 4 April 2020; Accepted: 27 April 2020; Published: 30 April 2020

check for updates

\begin{abstract}
Using the Global Navigation Satellite System (GNSS), it is difficult to provide continuous and reliable position service for vehicle navigation in complex urban environments, due to the natural vulnerability of the GNSS signal. With the rapid development of the sensor technology and the reduction in their costs, the positioning performance of GNSS is expected to be significantly improved by fusing multi-sensors. In order to improve the continuity and reliability of the vehicle navigation system, we proposed a multi-sensor tight fusion (MTF) method by combining the inertial navigation system (INS), odometer, and barometric altimeter with the GNSS technique. Different fusion strategies were presented in the open-sky, insufficient satellite, and satellite outage environments to check the performance improvement of the proposed method. The simulation and real-device tests demonstrate that in the open-sky context, the error of sensors can be estimated correctly. This is useful for sensor noise compensation and position accuracy improvement, when GNSS is unavailable. In the insufficient satellite context (6 min), with the help of the barometric altimeter and a clock model, the accuracy of the method can be close to that in the open-sky context. In the satellite outage context, the error divergence of the MTF is obviously slower than the traditional GNSS/INS tightly coupled integration, as seen by odometer and barometric altimeter assisting.
\end{abstract}

Keywords: GNSS; INS; odometer; barometric altimeter; clock model; tight fusion

\section{Introduction}

For vehicle navigation in urban environments, the Global Navigation Satellite System (GNSS) cannot provide continuous and reliable high-precision location services in cases where the transmitted signals are reflected and attenuated by trees and buildings, or even blocked in underground garages and tunnels. Fusing multi-sensors with GNSS is an effective way to improve the availability of the navigation system. The inertial measurement unit (IMU) is the suitable sensor for vehicles, with quickly improved performance and largely reduced cost of inertial sensors, and it is expected that by applying the high-grade IMUs to urban vehicles, the highly accurate clock model can help improve the performance of the GNSS in low satellite visibility conditions. Recently, the GNSS receivers have included a Temperature Compensated Crystal Oscillator (TCXO), characterized by a short-term stability $(\tau=1 \mathrm{~s})$ of $1 \times 10^{-9} \mathrm{~s}$ that leads to a $0.3 \mathrm{~m}$ error in the pseudorange. As the chip-scale atomic clock (CSAC) technology is developing rapidly, it can achieve a short-term stability $(\tau=1 \mathrm{~s})$ of $2.5 \times 10^{-12}$ s stability, about a $0.075 \mathrm{~m}$ error in the pseudorange [1]. The stability is comparable to the traditional Rubidium clock, and the size is just about one hundred milliliters. The atomic chip clock can be employed to improve the GNSS performance. The mileage data of the wheels can be 
obtained directly from the vehicle, which is helpful to reduce divergence in a long satellite outage environment. The barometric altimeter can provide elevation to distinguish the vehicle on or under the overpass in urban environments. The aforementioned sensors are expected to be widely applied in vehicle navigation in the near future, considering the advantage of easy acquisition for vehicles as well as their low cost.

The research on fusing the aforementioned sensors and GNSS can be mainly divided into two categories. The first is the GNSS/DR (Dead Reckoning sensor), like the GNSS/INS fusion, and the other is GNSS augmented with a barometric altimeter and clock model aiding. In case of the short-term failure of Global Positioning System (GPS) signals, INS can be used to determine position and orientation by integrating acceleration and angular velocity. Previous research has been carried out on three GNSS/INS integration types: the loosely coupled, tightly coupled, and ultra-tight integration [2-4]. The ultra-tight integration is suitable for highly dynamic cases or weak signal environments, but the mobility of vehicles is relatively low. In addition, the integration needs to adjust the receiver tracking loop, and it is not accessible when using commercial receivers. The main difference between loosely and tightly coupled integration is the measurement type. For loosely coupled integration, the measurements are the position and velocity, while they are pseudoranges and doppler shifts for the tightly coupled integration. The tightly coupled integration is more suitable in case of reduced satellite signal availability [5-8]. Recently, as raw observations retrieved from the GNSS chipsets in smart devices are now available to the users and developers, a number of studies on low-cost, tightly coupled GNSS/INS have also been carried out, and they were found to have good performance $[9,10]$. However, the position error of INS accumulates rapidly during the period of GNSS outage, so that the GNSS/INS fusion cannot provide position service in the indoor environment for a long time.

The odometer is added to the GNSS/INS system to solve the problem of the quadratic growing for position error, when there is a long satellite outage. The velocity of vehicles can be obtained by the odometer, and the position is calculated by one integration of velocity [11]. The divergence of the odometer is slower than INS. The GPS/IMU/odometer integration systems were designed using different filters to improve the position performance of the system. Falco et al. designed a GPS/IMU/odometer tightly coupled system through the use of a Kalman Filter (KF) [12]. Liu also used the KF to integrate GPS, BDS (BeiDou Navigation Satellite System), MEMS-INS (Micro-Electro-Mechanical System-Inertial Navigation System), and odometer sensors for real-time high-precision vehicle positioning in an urban degraded and denied environment [13]. Georgy designed a GPS/IMU/odometer integration system using a mixture particle filter [14]. Zengke designed a GPS/INS/odometer system based on a Fuzzy Neural Network (FNN), using odometer velocity correction to enhance resolution accuracy over a long GPS outage [15]. The test results report that the odometer can help to increase the positioning performance effectively, and the KF is more suitable for practical applications due to its low complexity and good performance. However, there are two disadvantages for the GPS/INS/odometer system: one is the quick height divergence when there is a satellite outage, and the other is that the fusion algorithms only consider two cases with and without satellites, and the insufficient satellites case is not specially treated and improved.

The barometric altimeter can help to solve the above problems. The local height can be calculated by the relationship between local pressure and reference pressure, which is generally standard atmosphere. The standard atmosphere is the mean sea-level atmospheric pressure, where the height is zero. The standard atmosphere is different in various regions, and changes over time. Parviainen et al. calculates the user's altitude by a differential way in personal navigation [16]. This method needs to set up a reference station, and its baseline is not very long. Yen treats elevation as a geocentric satellite and combines it with available GPS to estimate position in the GNSS signal-degraded environment [17]. The research of combining barometric with GPS/INS/odometer is rather limited. Chiang et al. simply treats the barometer height as a measurement in the KF in the INS/GNSS/odometer/barometer integration system [18], without using advantages of the barometer treated as a geocentric satellite in tightly coupled integration. Moreover, in this paper, the pressure at the initial position is treated as the 
reference pressure, and it is not a proper method, for the pressure tendency changes a lot when the vehicle is far from the initial position and also varies with time.

The quality of GNSS receiver clocks also exhibit high impacts on the performance of positioning in the complex environment $[19,20]$. Krawinkel assessed the benefits of receiver clock modeling in code-based GNSS navigation, and achieved position solutions with only three satellites in view [21]. $\mathrm{Li}$ employed chip-scale atomic clock (CSAC) to assist BDS positioning, and the clock prediction accuracy achieved $3 \mathrm{~ns}$ in $60 \mathrm{~min}$ [22]. These results show the improvement in GNSS position brought by a high-stability clock and analyze the influence of satellite geometry range on clock model-assisted positioning. However, the recent research mainly focuses on the clock model-assisted method in GNSS position, and few works discuss the clock model affection in the GNSS/INS integration system.

In urban navigation, the vehicle always travels along open-sky, insufficient satellites, and satellite outage environments [23]. Paul D points out that in challenging environments, the context is critical to the operation of a navigation or positioning system [24]. Saeedi uses low-cost sensors to improve the accuracy and robustness of a context-aware navigation system for pedestrian positioning, considering the person activity and placement context [25]. However, few public literatures focus on the context-based vehicle fusion position.

The purpose of this paper is to report a method systematically fusing the GNSS, INS, odometer, barometric, and clock model, named the multi-sensor tight fusion (MTF) method, for vehicle navigation in urban environments. It designs the different strategies for vehicles traveling along open-sky, insufficient satellites, and satellite outage contexts. In addition, to reduce the cost of setting up a reference station, the method compensates the error of the barometric altimeter when there are good satellite visibility conditions. Simulation and real-device test results show that the MTF method is significantly better than the traditional GNSS/INS tightly coupled integration in challenging urban environments. Following this introduction, the MTF method is presented along with the description of the observation model of all included sensors (GNSS, INS, odometer, barometric, and clock). The reference pressure is estimated by Kalman Filter (KF), to calculate the barometric height. The performance of the proposed MTF method is then validated in both simulated and real-device experiments under different environments. Finally, the summary and conclusions are given.

\section{Methods}

\subsection{Observation Model of Sensors}

The observation model of the GNSS, odometer, and barometric altimeter are described as follows. The GNSS pseudorange measurement, $\rho_{i}$, between satellite and receiver can be expressed as [26]

$$
\rho_{i}=\sqrt{\left(x-x^{i}\right)^{2}+\left(y-y^{i}\right)^{2}+\left(z-z^{i}\right)^{2}}+t_{u}
$$

where $x, y$, and $z$ are three-dimensional coordinates of the user position in the Earth-centered Earth-fixed (ECEF) coordinate system, and $t_{u}$ is the receiver clock offset. $x^{i}, y^{i}$, and $z^{i}$ are three-dimensional coordinates of the $i$-th satellite position in the ECEF coordinate system, and four or more satellites are needed to estimate the user's position. The pseudorange rate, $\dot{\rho}_{i}$, is given as follows [26].

$$
\dot{\rho}_{i}=e_{i x}\left(\dot{x}-\dot{x}^{i}\right)+e_{i y}\left(\dot{y}-\dot{y}^{i}\right)+e_{i z}\left(\dot{z}-\dot{z}^{i}\right)+d t_{u}
$$

with the notation $\dot{x}, \dot{y}$, and $\dot{z}$ for three-dimensional coordinates of the user velocity in the ECEF coordinate system, $d t_{u}$ for clock drift, and $\dot{x}^{i}, \dot{y}^{i}$, and $\dot{z}^{i}$ for three-dimensional coordinates of the i-th satellite velocity in the ECEF coordinate system. The line-of-sight vector, $e_{i}$, is expressed as $e_{i x}=\left(x-x^{i}\right) / r^{i}$, $e_{i y}=\left(y-y^{i}\right) / r^{i}, e_{i z}=\left(z-z^{i}\right) / r^{i}$, and $r^{i}=\sqrt{\left(x-x^{i}\right)^{2}+\left(y-y^{i}\right)^{2}+\left(z-z^{i}\right)^{2}}$. The observations of INS are position, velocity, and attitude and the calculation from IMU measurement, acceleration, and angular velocity can be viewed in Reference [27]. 
The odometer measurement is the vehicle velocity, $v_{O D O}$, in the body frame, and the observation equation is $[14,15]$

$$
v_{\mathrm{ODO}}=s f_{\mathrm{ODO}} \cdot C_{n}^{b} \cdot v^{n}{ }_{3 \times 1}
$$

where the forward velocity of $v_{O D O}$ is measured by the odometer, and the lateral and up velocity of $v_{O D O}$ are supposed as zero, considering the Non-Holonomic Constraint (NHC). $v^{n}{ }_{3 \times 1}$ is the velocity of user in the local frame, $C_{n}^{b}$ is the rotation matrix from the local frame to the body frame, and $s f_{O D O}$ is the scale factor.

The measurement of the barometric altimeter is local pressure, $P$, and differential Equation (4) describing the altitude, $H$, as a function of the local pressure, $P$, using the isothermal atmospheric model of the US Standard Atmosphere [28,29].

$$
H-H_{0}=\frac{R T}{g} \ln \frac{P_{0}}{P}=18,410 *\left(1+\frac{t}{273.15}\right) * \lg \frac{P_{0}}{P}
$$

when $H_{0}$ is equal to zero, $P_{0}$ is the standard atmospheric pressure. The standard atmospheric pressure varies in different regions and changes continuously over time. In Equation (4), $R$ is the specific gas constant equal to $R^{*} / m, R^{*}$ is the ideal gas constant equal to $8.31446 \mathrm{~J} / \mathrm{K} / \mathrm{m}^{\circ} l, m$ is the average molecular weight equal to $28.9634 \mathrm{~g} / \mathrm{m}^{\circ} \mathrm{l}, g$ is the standard gravity value equal to $9.80665 \mathrm{~m} / \mathrm{s}^{2}$, and $t$ is the local temperature in the unit of Celsius.

In case the barometric altimeter is taken as a geocentric satellite, the observation equation can be written as [26]:

$$
\frac{x^{2}+y^{2}}{\left(R_{e}+h_{B A R O}\right)^{2}}+\frac{z^{2}}{\left(R_{p}+h_{B A R O}\right)^{2}}=1
$$

where $h_{B A R O}$ is the barometric measurement, and is calculated by Equation (4). The clock model measurement is the predicted clock error, $t_{\text {prediction, }}$ and the observation equation is

$$
t_{\text {prediction }}=t_{u}
$$

with the notation $R_{e}$ for the semi-major axis of earth, and $R_{p}$ for the semi-minor axis.

\subsection{Fusion Method Design}

The proposed MTF method is illustrated in Figure 1. It uses a sequential Extended Kalman Filter (EKF), and the EKF is widely applied in practical projects [12]. The MTF method considers the observation model mentioned in Section 2.1 to design the KF. In the KF, the measurements of each sensor update sequentially. Based on traditional GNSS/INS tightly coupled integration, the MTF method adds the odometer and barometric altimeter sensor to the filter. The MTF method designs different strategies for distinct environments, such as the open-sky, insufficient satellite, satellite outage cases. In the open-sky context, the KF carries out altimeter and odometer measurement updates by turning on switches 3 and 4, indicated by the red circle in Figure 1. As the accuracy of GNSS is high, the errors of sensors can be estimated correctly, which are used to compensate the measurement and improve position performance when the GNSS signal is impaired. In the insufficient satellite context, the KF turns on switches 1 and 2, and turns off switches 3 and 4 . The barometric altimeter, treated as a geocentric satellite, is used for measurement updates with the remaining satellites and clock model. The clock model is used to predict the clock error. In the satellite outage case, the KF turns on switches 3 and 4 , and turns off switches 1 and 2 . The odometer and barometric altimeter are used to reduce the horizontal and height position error divergence, respectively. 


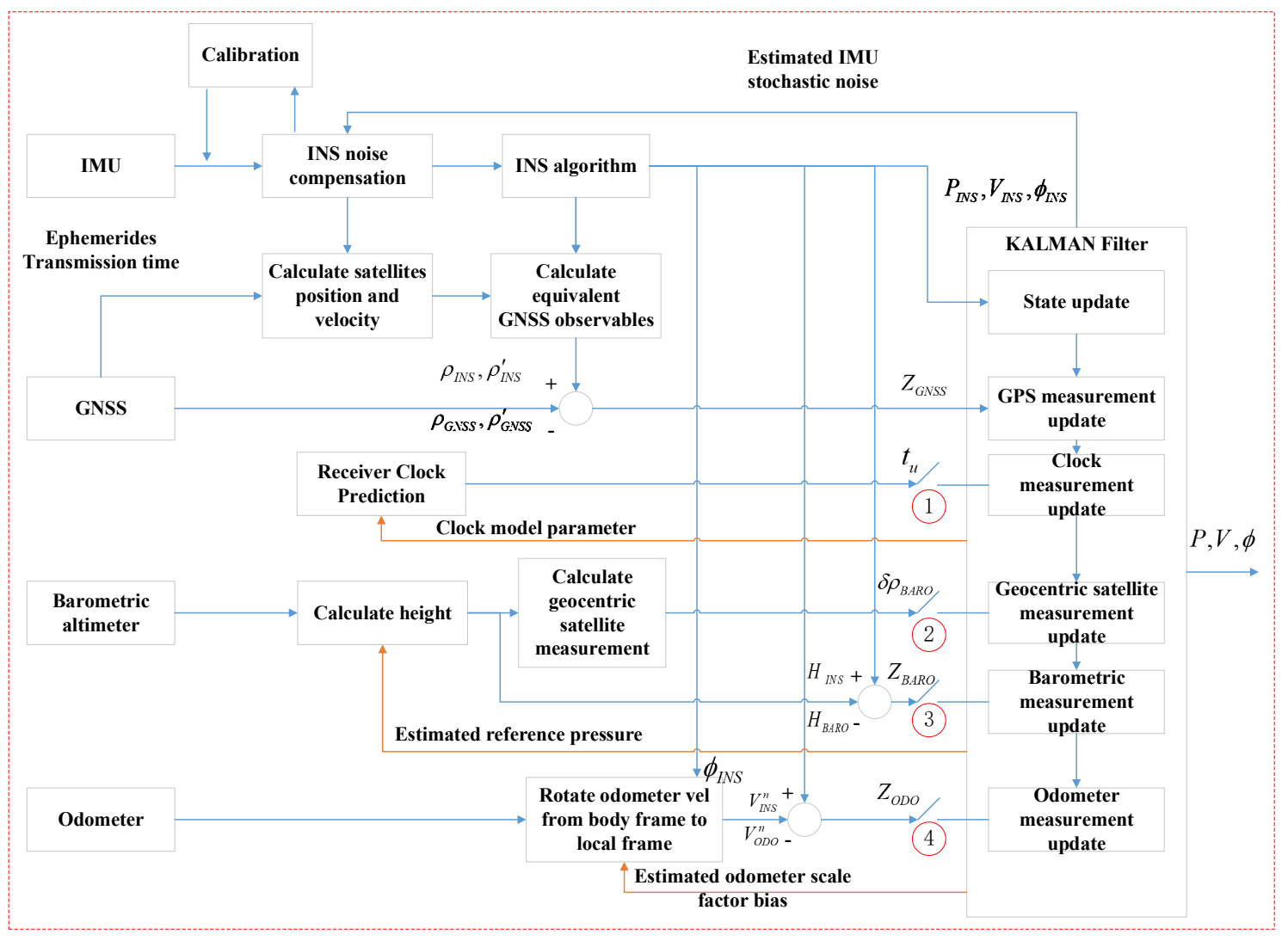

Figure 1. Flow chart of the multi-sensor tight fusion (MTF) method.

In Part I of Figure 1, for the IMU, the block labeled as INS noise compensation is used to compensate the bias of the accelerometer and gyro. The INS algorithm processes the inertial navigation solution, which can calculate position, velocity, and altitude.

For the GNSS, the block labeled as calculate equivalent GNSS observables is used to calculate the equivalent pseudorange and pseudorange rate using the position, velocity of the satellite, and INS. The pseudorange and the pseudorange rate difference between GNSS and INS is the KF measurement.

For the odometer, the scale factor is used to compensate the forward velocity measurement. The velocity difference between the odometer and INS in the local frame is the KF measurement.

For the barometric altimeter, calculate height calculates the local height by the relationship between local pressure and reference pressure, which is generally standard atmosphere. The standard atmosphere is the mean sea-level atmospheric pressure, and where the height is zero. In the MTF method, the reference pressure is estimated by the KF. The height difference between barometric height and INS is the KF measurement. In addition, the barometric height also can be used to calculate the geocentric satellite measurement, which is used in the insufficient satellite context.

\subsection{Fusion Filter Design}

In the KF, the dynamic state-space linear model is expressed as [12,27]

$$
\begin{aligned}
& \delta \dot{x}=\phi \cdot \delta x+v \\
& Z=H \cdot \delta x+e
\end{aligned}
$$

where notation $\delta x$ is for the state, $v$ is for the process noise, $\phi$ is for the system transition matrix, $Z$ is for the KF measurement, $H$ is for the measurement matrix, and $e$ is for the measurement noise. The filter 
state $\delta x$ includes the system state and the error of sensors. The system states are altitude, velocity, and position.

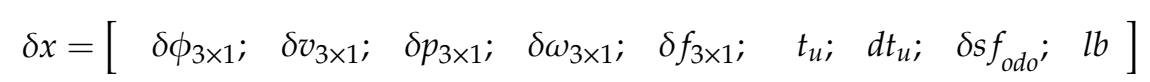

where, $\delta \phi_{3 \times 1}$ is the altitude error vector in the local frame, $\delta v_{3 \times 1}$ is the velocity error vector in the local frame, $\delta p_{3 \times 1}$ is the position error vector in the local frame, $\delta \omega_{3 \times 1}$ is the error vector related to the angular rates measured by the IMU in the body frame, $\delta f_{3 \times 1}$ is the error vector related to the specific forces measured by the IMU in the body frame, $t_{u}$ is the receiver's clock bias, where the unit is meters, $d t_{u}$ is the receiver's clock drift, where the unit is meters/second, $\delta s f_{\text {odo }}$ is the error related to the odometer scale factor, and $l b$ is the estimated reference atmospheric pressure.

$$
\phi=\left[\begin{array}{ccccccccc}
M_{a a} & M_{a v} & M_{a p} & -C_{b}^{n} & 0_{3 \times 3} & 0_{3 \times 1} & 0_{3 \times 1} & 0_{3 \times 1} & 0_{3 \times 1} \\
M_{v a} & M_{v v} & M_{v p} & 0_{3 \times 3} & C_{b}^{n} & 0_{3 \times 1} & 0_{3 \times 1} & 0_{3 \times 1} & 0_{3 \times 1} \\
0_{3 \times 3} & M_{p v} & M_{p p} & 0_{3 \times 3} & 0_{3 \times 3} & 0_{3 \times 1} & 0_{3 \times 1} & 0_{3 \times 1} & 0_{3 \times 1} \\
0_{3 \times 3} & 0_{3 \times 3} & 0_{3 \times 3} & -\operatorname{diag}\left(1 / \tau_{g 1 \times 3}\right) & 0_{3 \times 3} & 0_{3 \times 1} & 0_{3 \times 1} & 0_{3 \times 1} & 0_{3 \times 1} \\
0_{3 \times 3} & 0_{3 \times 3} & 0_{3 \times 3} & 0_{3 \times 3} & -\operatorname{diag}\left(1 / \tau_{a 1 \times 3}\right) & 0_{3 \times 1} & 0_{3 \times 1} & 0_{3 \times 1} & 0_{3 \times 1} \\
0_{1 \times 3} & 0_{1 \times 3} & 0_{1 \times 3} & 0_{1 \times 3} & 0_{1 \times 3} & 0 & 1 & 0 & 0 \\
0_{1 \times 3} & 0_{1 \times 3} & 0_{1 \times 3} & 0_{1 \times 3} & 0_{1 \times 3} & 0 & 0 & 0 & 0 \\
0_{1 \times 3} & 0_{1 \times 3} & 0_{1 \times 3} & 0_{1 \times 3} & 0_{1 \times 3} & 0 & 0 & 0 & 0 \\
0_{1 \times 3} & 0_{1 \times 3} & 0_{1 \times 3} & 0_{1 \times 3} & 0_{1 \times 3} & 0 & 0 & 0 & 0
\end{array}\right]
$$

where notation $\tau_{a 1 \times 3}$ and $\tau_{g 1 \times 3}$ represent the time constant vectors of the Gauss-Markov noises related to the accelerometers and gyroscopes, respectively. The $M$ matrixes are shown in the equations in the Appendix A.

The GNSS observation model is linearized through the first-order Taylor expansion, then the measurement vector and the measurement matrix are expressed as

$$
\begin{gathered}
Z_{G N S S}=\left[\begin{array}{c}
\rho_{I N S}^{1}-\rho_{G N S S}^{1} \\
\rho_{I N S}^{2}-\rho_{G N S S}^{2} \\
\cdots \\
\rho_{I N S}^{n}-\rho_{G N S S}^{n}
\end{array}\right] \\
H_{G N S S}=\left[\begin{array}{cccc}
\frac{x-x^{1}}{r_{1}} & \frac{y-y^{1}}{r_{1}} & \frac{z-z^{1}}{r_{1}} & 1 \\
\frac{x-x^{2}}{r_{2}} & \frac{y-y^{2}}{r_{2}} & \frac{z-z^{2}}{r_{2}} & 1 \\
\cdots & \cdots & \cdots & 1 \\
\frac{x-x^{n}}{r_{n}} & \frac{y-y^{n}}{r_{n}} & \frac{z-z^{n}}{r_{n}} & 1
\end{array}\right]
\end{gathered}
$$

using the notation $n$ for the number of satellites. The odometer observation model is linearized through the first-order Taylor expansion, then the measurement vector and the measurement matrix can be written as

$$
\begin{gathered}
Z_{O D O}=s f_{O D O} \cdot v_{I N S}^{n}-C_{b}^{n} v_{O D O} \\
H_{O D O}=\left[\begin{array}{llllllll}
-v_{O D O} \times & \operatorname{diag}\left(s f_{O D O}\right)_{3 \times 3} & 0_{3 \times 3} & 0_{3 \times 3} & 0_{3 \times 3} & 0_{3 \times 2} & \left(v_{I N S}^{n}\right)_{3 \times 1} & 0_{3 \times 1}
\end{array}\right]
\end{gathered}
$$

The barometric altimeter observation model is linearized through the first-order Taylor expansion, then the measurement and the measurement matrix are expressed as:

$$
\begin{aligned}
& Z_{\text {BARO }}=H_{I N S}-\left(H_{0}+18,400 *\left(1+\frac{t}{273.15}\right) * \lg \frac{P_{0}}{P}\right)
\end{aligned}
$$

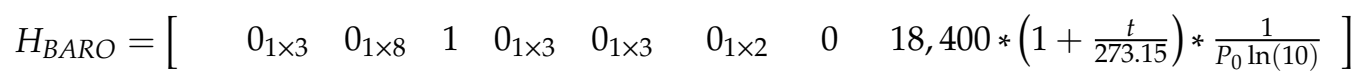


The GNSS/barometric altimeter/clock observation models are linearized through the first-order Taylor expansion, then the measurement and the measurement matrix are expressed as

$$
\begin{aligned}
& Z_{\text {sat } 2}=\left[\begin{array}{llll}
\delta \rho^{1} & \delta \rho^{2} & \delta \rho_{\text {BARO }} & t_{\text {prediction }}
\end{array}\right]^{T} \\
& \delta \rho_{\text {BARO }}=\frac{1}{2}\left(\frac{x_{I N S}^{2}+y_{I N S}^{2}}{\left(R_{e}+h_{B A R O}\right)^{2}}+\frac{z_{I N S}^{2}}{\left(R_{p}+h_{B A R O}\right)^{2}}-1\right) \\
& H_{\text {sat } 2}=\left[\begin{array}{lllllllll} 
& 0_{4 \times 3} & 0_{4 \times 3} & M_{1} * C_{n}^{e} & 0_{4 \times 3} & 0_{4 \times 3} & M_{2} & 0_{4 \times 1} & 0_{4 \times 2}
\end{array}\right] \\
& M_{1}=\left[\begin{array}{ccc}
\frac{x-x^{1}}{r_{1}} & \frac{y-y^{1}}{r_{1}} & \frac{z-z^{1}}{r_{1}} \\
\frac{x-x^{2}}{r_{2}} & \frac{y-y^{2}}{r_{2}} & \frac{z-z^{2}}{r_{2}} \\
\frac{x}{\left(R_{e}+h_{B A R O}\right)^{2}} & \frac{y^{2}}{\left(R_{e}+h_{B A R O}\right)^{2}} & \frac{z}{\left(R_{p}+h_{B A R O}\right)^{2}} \\
0 & 0 & 0
\end{array}\right] \\
& M_{2}=\left[\begin{array}{llll}
1 & 1 & 0 & 1
\end{array}\right]^{T}
\end{aligned}
$$

where $\delta \rho^{1}$ is the GNSS measurement, the same as in Equation (11), $t_{\text {prediction }}$ is the predicted clock error, and $C_{n}^{e}$ is the rotation matrix from the local frame to the ECEF frame.

\section{Simulation Test}

To validate the proposed MTF method, a vehicle traveling scenario is simulated, and the associated sensor measurements, i.e., IMU, GNSS, odometer, and barometric altimeter, are generated accordingly. As the sensor errors are known in advance in the simulation, they can be used to evaluate the estimated sensor errors (including accelerometer bias, gyroscope bias, odometer scale factor, reference pressure of barometric altimeter, and GNSS clock model) in the KF in the open-sky case. Only if the estimated sensor errors converge to the right value soon will the position in insufficient satellite and satellite outage cases perform well.

The actions of vehicles include stationary, uniform speed, acceleration, deceleration, turning, climbing, and downhill. The total simulation period covers $3664 \mathrm{~s}$. The simulated trajectory is shown in Figure 2, where the red star is the start point. The initial position, velocity, and altitude are given in Table 1. GNSS measurements are pseudoranges and pseudorange rates, which are computed by combing the position and velocity of the vehicle with that of satellites, obtained from GPS ephemeris on 21 November 2019. The Gaussian noise of $1 \mathrm{~m}$ is added to the pseudorange, and the Gaussian noise of $0.1 \mathrm{~m} / \mathrm{s}$ is added to the pseudorange rate. The clock model is also added to the pseudorange and pseudorange rate. The clock drift is constant at $1 \times 10^{-8}$, which is equivalent to 2.99792458 $\mathrm{m} / \mathrm{s}$, and the Gaussian noise of $1 \times 10^{-11}$ is added to the clock drift. The initial clock error is zero. The IMU measurements are the specific force and angular rate from the accelerometer and gyroscope, respectively. The bias of $100 \mathrm{ug}$ and velocity random walk of $10 \mathrm{ug} / \sqrt{\mathrm{Hz}}$ is added to the specific force. The bias of $0.05^{\circ} / \mathrm{hr}$ and velocity random walk of $1 \times 10^{-3} / \sqrt{\mathrm{hr}}$ is added to the angular rate. The odometer measurement is forward velocity, and its scale factor is 0.9 . The Gaussian noise of $0.1 \mathrm{~m} / \mathrm{s}$ is added to the velocity. The barometric altimeter measurement is local pressure, and reference pressure is $1000 \mathrm{hPa}$, where the height is zero. The Gaussian noise of $0.1 \mathrm{hPa}$ is added to local pressure. Table 2 lists the simulation parameters of each sensor. 

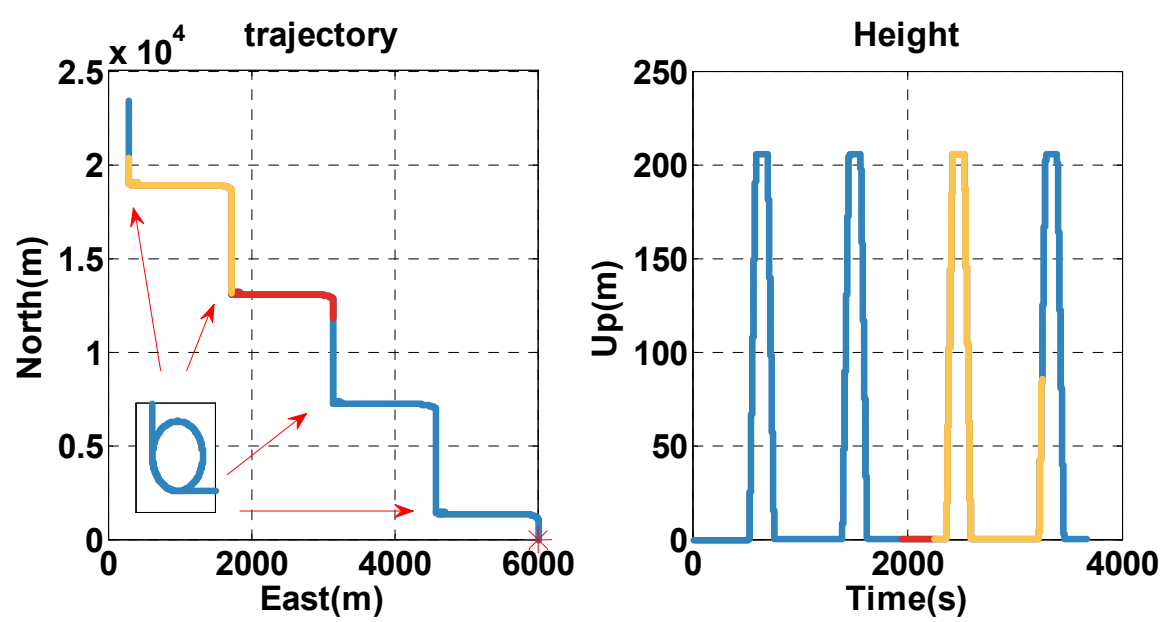

Figure 2. Horizontal trajectory and height of the simulation test. The blue, red, and yellow lines indicate the open-sky, insufficient satellite, and satellite outage context, respectively.

Table 1. Initial state parameters of the trajectory.

\begin{tabular}{cc}
\hline Type & Initial State of the Trajectory \\
\hline Lan/Lon/Height & $34.246048^{\circ}, 108.909664^{\circ}, 380 \mathrm{~m}$ \\
Heading/Roll/Yaw & $0^{\circ}, 0^{\circ}, 0^{\circ}$ \\
East/North/Up velocity & $0 \mathrm{~m} / \mathrm{s}, 0 \mathrm{~m} / \mathrm{s}, 0 \mathrm{~m} / \mathrm{s}$ \\
\hline
\end{tabular}

Table 2. List of sensor simulation parameters.

\begin{tabular}{|c|c|c|}
\hline Sensor & Sensor Error & $\begin{array}{l}\text { Sample } \\
\text { Rate }\end{array}$ \\
\hline GNSS & $\begin{array}{l}\text { Pseudorange: Gaussian noise of } 1 \mathrm{~m} \text {. } \\
\text { Pseudorange rate: Gaussian noise of } 0.1 \mathrm{~m} / \mathrm{s} \text {. } \\
\text { Clock model: the clock drift is } 1 \times 10^{-8} \text {, and Gaussian noise of } 1 \times 10^{-11} \\
\text { is added to the clock drift. The initial clock error is zero. }\end{array}$ & $1 \mathrm{~Hz}$ \\
\hline Accelerometer & Bias of $100 \mathrm{ug}$, velocity random walk of $10 \mathrm{ug} / \sqrt{\mathrm{Hz}}$ & $100 \mathrm{~Hz}$ \\
\hline Gyroscope & Bias of $0.05^{\circ} / \mathrm{hr}$, angular random walk of $1 \times 10^{-3^{\circ}} / \sqrt{\mathrm{hr}}$ & $1 \mathrm{~Hz}$ \\
\hline Odometer & Scale factor is 0.9, Gaussian noise of $0.1 \mathrm{~m} / \mathrm{s}$ & $1 \mathrm{~Hz}$ \\
\hline $\begin{array}{l}\text { Barometric } \\
\text { altimeter }\end{array}$ & $\begin{array}{l}\text { Reference pressure is } 1000 \mathrm{hPa} \text { when the height is zero, Gaussian noise } \\
\text { of } 0.1 \mathrm{hPa} \text { (equivalent to about } 1 \mathrm{~m} \text { ) }\end{array}$ & $1 \mathrm{~Hz}$ \\
\hline
\end{tabular}

In the simulation test, the vehicle is driven along the contexts, such as open-sky, insufficient satellite, and satellite outage. To analyze the performance of the MTF method in the above contexts, the test time is divided into: during 0-1949 and 3251-3664 s, the vehicle is in the open-sky area, where the GNSS exhibits a high accuracy. The experiment analysis focuses on the error states of each sensor estimated by the filter, and that is significant for position resolution when the GNSS signal is blocked. During 1950-2249 s, the vehicle is in the area of the insufficient satellite, where the number of visible satellites decrease to 2. In Figure 3, the magenta dots indicate the remaining satellites at $1950 \mathrm{~s}$. The MTF method achieves measure-up by combining the remaining satellites with the barometer altimeter and the clock model. The analysis is conducted in terms of position and velocity errors comparing the proposed MTF method and the traditional GNSS/INS tightly coupled integration. During 2250-3250 s, the vehicle is in the satellite outage area. The MTF method carries on dead reckoning based on INS, odometer, and barometric altimeter. The performance of the MTF method is also compared with traditional GNSS/INS tightly coupled integration. 


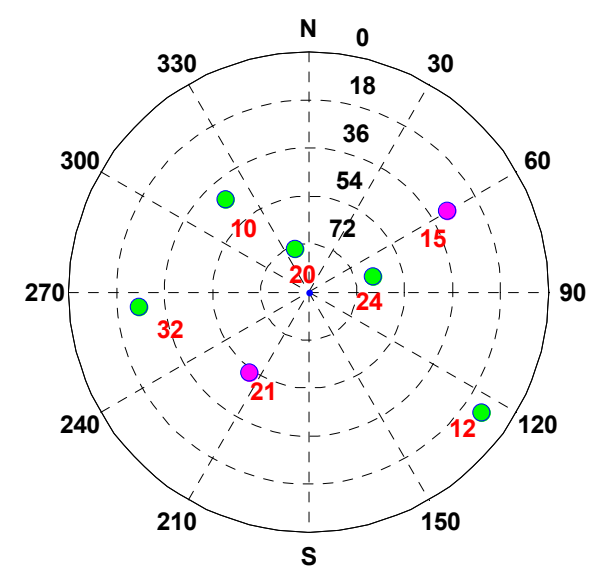

Figure 3. The sky plot of satellites for the epoch 1950 s, in which the green and magenta dots denote the blocked and visible satellites, respectively.

The noise parameters used in the filter are set as follows. The process noise is set as $1 \times 10^{-3^{\circ}} / \sqrt{\mathrm{hr}}$ for the altitude error state and $10 \mathrm{ug} / \sqrt{\mathrm{Hz}}$ for the velocity error state. The measurement noises are $1 \mathrm{~m}$ and $0.1 \mathrm{~m} / \mathrm{s}$ for GNSS pseudorange and pseudorange rate, $0.1 \mathrm{~m} / \mathrm{s}$ for odometer velocity, $1 \mathrm{~m}$ for barometric height, and $2.99 \times 10^{-3} \mathrm{~m}$ for the clock model, respectively.

\subsection{Open-Sky Case}

In the open-sky context (0-1949 s), the position and velocity errors of the MTF method are shown in Figure 4. The position and velocity errors are presented in the east, north, and up directions, and in the first $100 \mathrm{~s}$, the vehicle is stationary. The position and velocity errors converge at the beginning. However, the attitude errors decrease quickly when the vehicle starts to move. To statistically analyze the performance of the proposed MTF method, the errors are characterized by the 67th percentile. The orientation error is $0.46 \mathrm{~min}$, and the pitch and roll errors are $0.94 \mathrm{~s}$ and $0.90 \mathrm{~s}$. The horizontal position error is $0.22 \mathrm{~m}$, and the height error is $0.20 \mathrm{~m}$. The horizontal velocity error is $0.005 \mathrm{~m} / \mathrm{s}$, and the vertical velocity error is $0.0015 \mathrm{~m} / \mathrm{s}$. The performance of the fusion system is much better than GNSS alone by filtering out the noise on the pseudorange and the pseudorange rate.
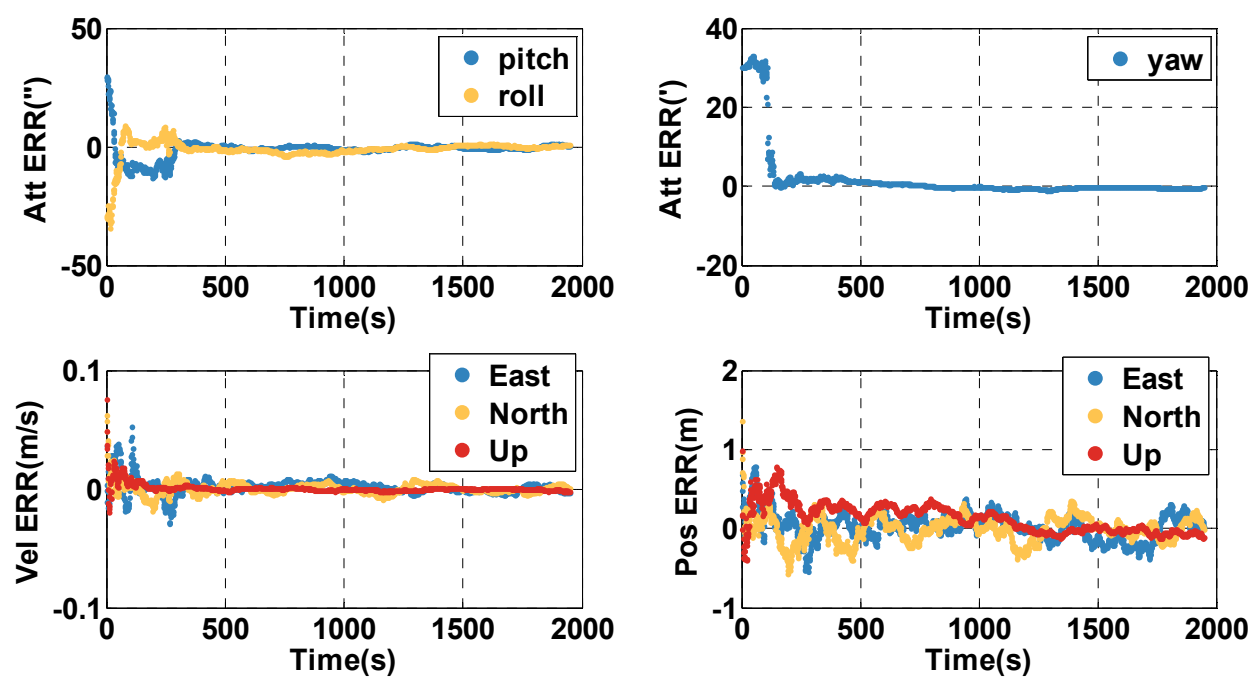

Figure 4. The position (Pos), velocity (Vel), and attitude (Att) errors (ERR) in the open-sky case by applying the MTF method.

Supposing the INS noise compensation module in Figure 1 stops working, the estimated biases of the gyro and accelerometer are shown in Figure 5. For the gyro, the errors in the right and forward 
direction, which is related to horizontal altitude, converge to the final value $(0.05$ degree/s shown in Table 2) in about $250 \mathrm{~s}$. The convergence time of error in the up direction is much longer, about $1000 \mathrm{~s}$, which is related to orientation. For the accelerometer, the error in the up direction, which is related to height, converges to the final value (100 ug, shown in Table 2$)$ in about $100 \mathrm{~s}$. The convergence time of errors in the right and forward direction is much longer, about $260 \mathrm{~s}$, which is related to horizontal movement.
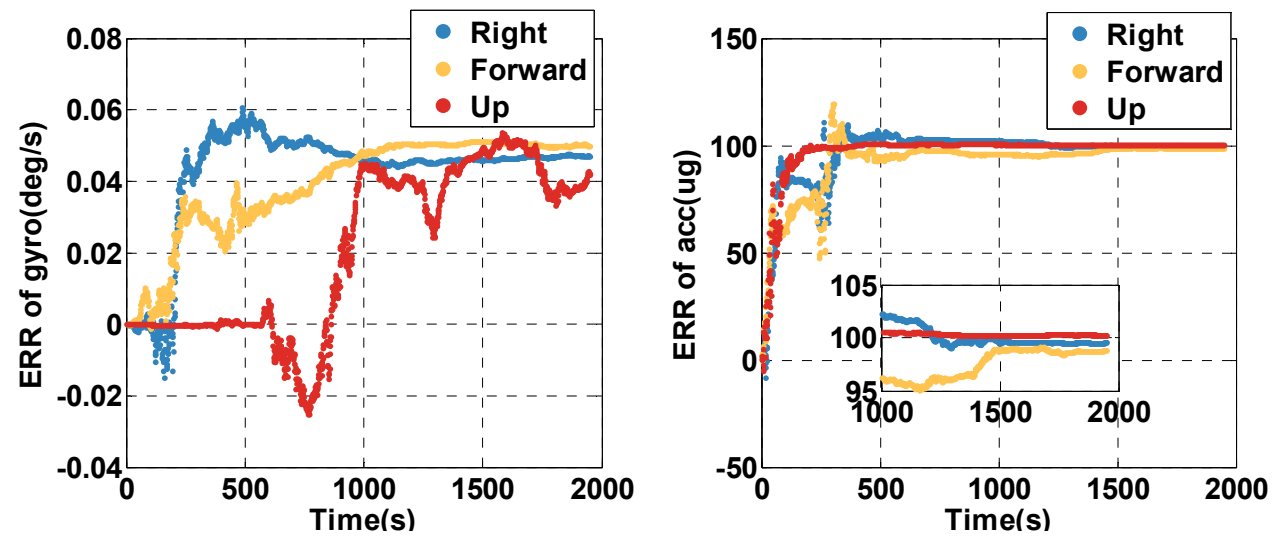

Figure 5. The estimated angular rate and specific force bias of gyro and accelerometer (acc) in the open-sky case. ERR = Error.

The estimated clock drift, error of the odometer's scale factor, and reference pressure are shown in Figure 6. The estimated clock drift quickly converges to the final value $(2.9979 \mathrm{~m} / \mathrm{s}$, shown in Table 2). The estimated scale factor error of the odometer converges to the final value $(-0.1)$, consistent with the scale factor in Table 2. The reference pressure converges to the final value (1000 hpa, shown in Table 2). Therefore, the sensor errors can be estimated correctly in the open-sky context.
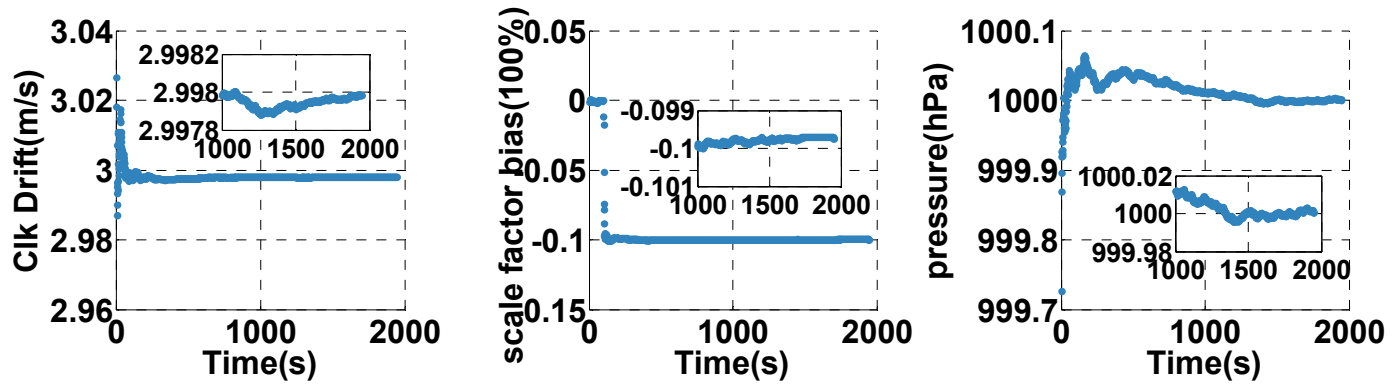

Figure 6. The estimated clock (Clk) drift, error of the odometer' scale factor, and reference pressure in the open-sky case.

\subsection{Insufficient Satellite Case}

The vehicle travel in the insufficient satellite context lasts for 6 min (1950-2249 s). As shown in Figure 3, the remaining visible satellites are presented in red circles for the epoch of $1950 \mathrm{~s}$. The position and velocity errors of the MTF method in the east, north, and up directions are shown in Figure 7. The errors of the traditional GNSS/INS tightly coupled integration are shown in Figure 8. The errors of the proposed method do not grow over 6 min in Figure 7, but the errors of GNSS/INS tightly coupled integration increase in Figure 8, especially the position error. In the same time, Figure 7 shows that the error of the MTF method is unstable at the beginning of the period, and the reason is that the new measurements update causes slight fluctuation in the filter. The error statistics are shown in Table 3, and they are characterized by the 95th percentile. Comparing the MTF method with GNSS/INS, the horizontal position errors of the two methods are approximately $0.3417 \mathrm{~m}$ against $0.6999 \mathrm{~m}$, the height errors are approximately $0.1851 \mathrm{~m}$ against $0.5227 \mathrm{~m}$, the horizontal velocity errors 
are approximately $0.0056 \mathrm{~m} / \mathrm{s}$ against $0.0087 \mathrm{~m} / \mathrm{s}$, and the vertical velocity errors are approximately $0.0014 \mathrm{~m} / \mathrm{s}$ against $0.0015 \mathrm{~m} / \mathrm{s}$. The accuracy of the MTF method is close to that in the open-sky context, and performs better than GPS/INS tightly coupled integration.
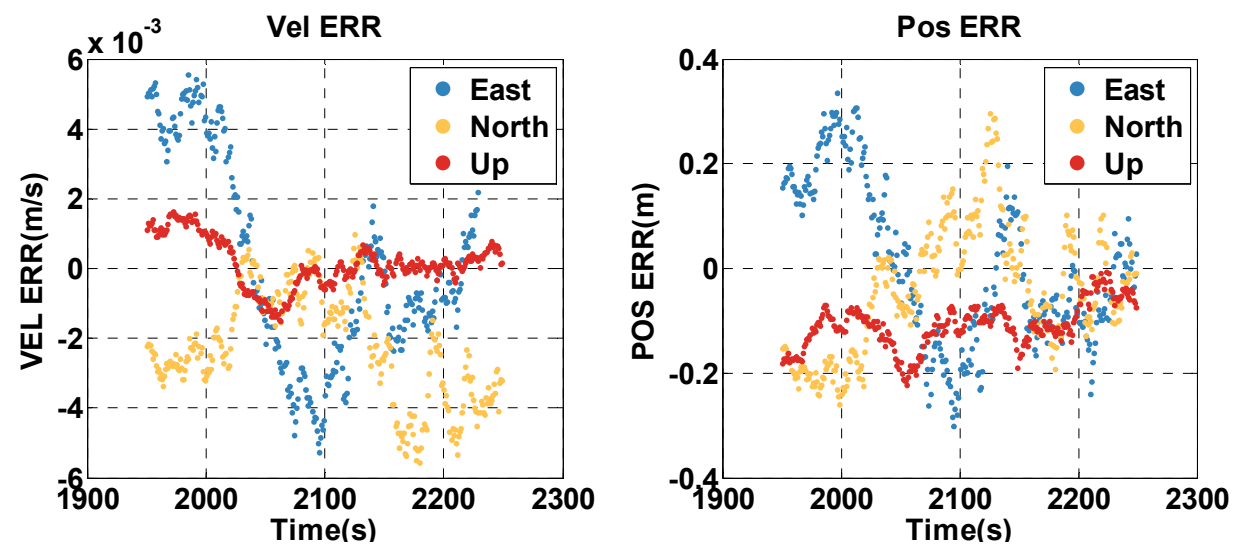

Figure 7. The position and velocity errors of the MTF method in the insufficient satellite case.
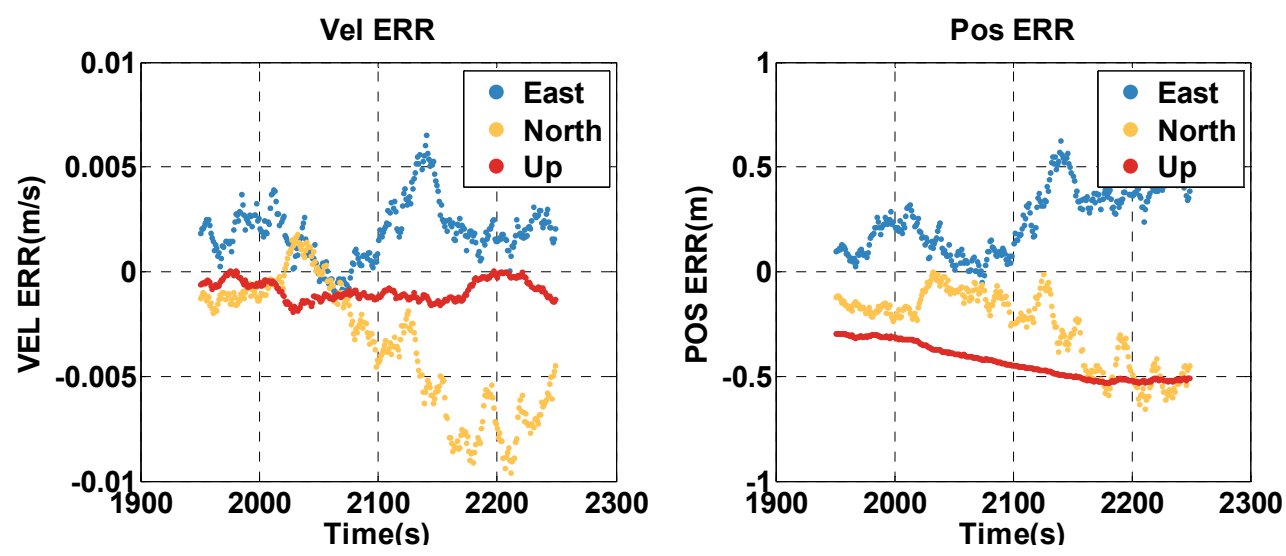

Figure 8. The position and velocity errors of the traditional GNSS/INS tightly coupled integration solution in the insufficient satellite case.

Table 3. Statistical analysis of the errors of the MTF method and the traditional GNSS/INS tightly coupled integration in the insufficient satellites context in the simulation test.

\begin{tabular}{ccccc}
\hline Method & $\begin{array}{c}\text { Horizontal } \\
\text { Velocity by 95th } \\
\text { Percentile }(\mathbf{m} / \mathbf{s})\end{array}$ & $\begin{array}{c}\text { Vertical Velocity } \\
\text { by 95th Percentile } \\
(\mathbf{m} / \mathbf{s})\end{array}$ & $\begin{array}{c}\text { Horizontal } \\
\text { Position by 95th } \\
\text { Percentile }(\mathbf{m})\end{array}$ & $\begin{array}{c}\text { Height by 95th } \\
\text { Percentile }(\mathbf{m})\end{array}$ \\
\hline MTF & 0.0056 & 0.0014 & 0.3417 & 0.1851 \\
GNSS/INS & 0.0087 & 0.0015 & 0.6999 & 0.5227 \\
\hline
\end{tabular}

\subsection{Satellite Outage Case}

The vehicle travels in the satellite outage context for about 16 mins (2250-3250 s). The position and velocity errors of the MTF method are shown in Figure 9. The errors of the traditional GNSS/INS tightly coupled integration are shown in Figure 10. The errors of both methods increase over time, but that of the MTF method is much slower. In addition, the MTF height error does not grow over time. The error statistics are shown in Table 4. The errors are characterized by the 95th percentile. Comparing the MTF method with GNSS/INS, the horizontal position errors of the two methods are approximately $15.04 \mathrm{~m}$ against $49.76 \mathrm{~m}$, the height errors are approximately $0.214 \mathrm{~m}$ against $5.713 \mathrm{~m}$, the horizontal velocity errors are approximately $0.0332 \mathrm{~m} / \mathrm{s}$ against $0.1337 \mathrm{~m} / \mathrm{s}$, and the vertical velocity 
errors are approximately $0.0017 \mathrm{~m} / \mathrm{s}$ against $0.0147 \mathrm{~m} / \mathrm{s}$. The MTF method has obvious advantages in both the horizontal and height direction by the aiding of the odometer and barometric altimeter.
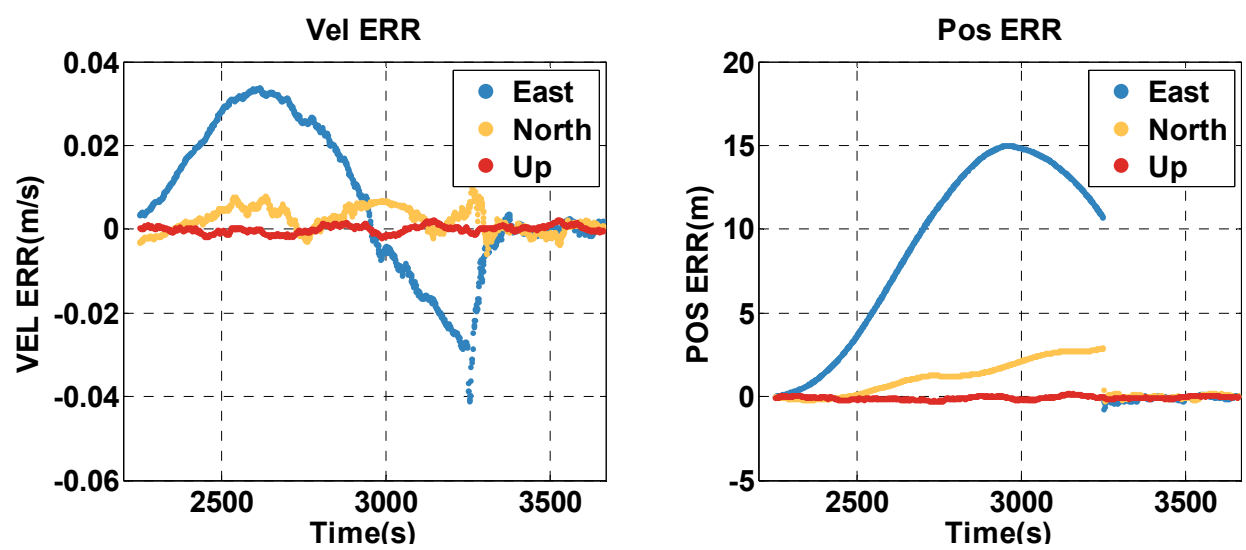

Figure 9. The position and velocity errors of the proposed MTF method in the satellite outage context.
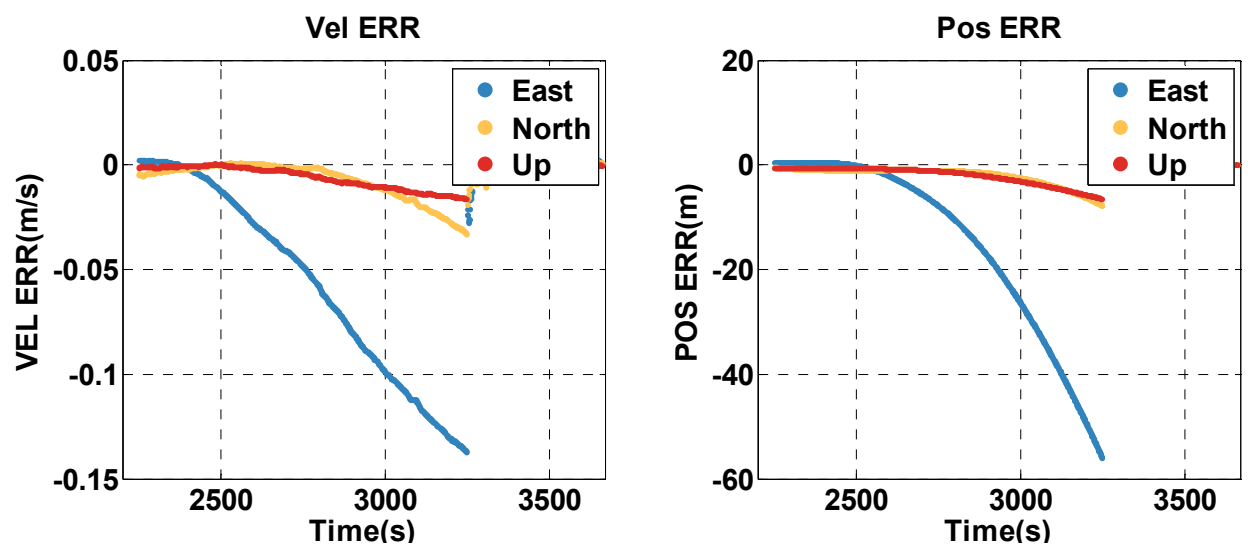

Figure 10. The position and velocity errors of the traditional GNSS/INS tightly coupled integration in the satellite outage case.

Table 4. Comparison of statistical results generated by the proposed MTF method and conventional GNS/INS coupled integration in the satellite outage case (the 95th percentile).

\begin{tabular}{ccccc}
\hline Method & $\begin{array}{c}\text { Horizontal } \\
\text { Velocity by 95th } \\
\text { Percentile (m/s) }\end{array}$ & $\begin{array}{c}\text { Vertical Velocity } \\
\text { by 95th Percentile } \\
(\mathbf{m} / \mathbf{s})\end{array}$ & $\begin{array}{c}\text { Horizontal } \\
\text { Position by 95th } \\
\text { Percentile (m) }\end{array}$ & $\begin{array}{c}\text { Height by 95th } \\
\text { Percentile (m) }\end{array}$ \\
\hline MTF & 0.0332 & 0.0017 & 15.04 & 0.214 \\
GNSS/INS & 0.1337 & 0.0147 & 49.76 & 5.713 \\
\hline
\end{tabular}

\section{Real-Device Test}

In the experiment test, the sensor measurements are obtained by a test platform in Figure 11, that consists of the GNSS receiver (Novatel ProPak6 receiver and Novatel GPS-704 antenna), IMU (gyro bias stability: $0.5^{\circ} / \sqrt{\mathrm{hr}}$, angular random walk: $0.012^{\circ} / \sqrt{\mathrm{hr}}$; accelerometer bias stability: $1250 \mathrm{ug}$, velocity random walk: $100 \mathrm{ug} / \sqrt{\mathrm{Hz}}$ ), barometric altimeter (VAISALA PTU 301, resolution: $0.01 \mathrm{hPa}$ ), and an odometer (1000 pulse per wheel round). The antenna of the GNSS is mounted on board a vehicle, and the odometer is on the right rear wheel. The sensor measurements are collected on the laptop, and Real-Time Kinematic (RTK) + Strap-down Inertial Navigation System (SINS) results are processed by Inertial Explorer (IE) Post-Processing software (Novatel) are used as a highly precise reference to evaluate the MTF method. 


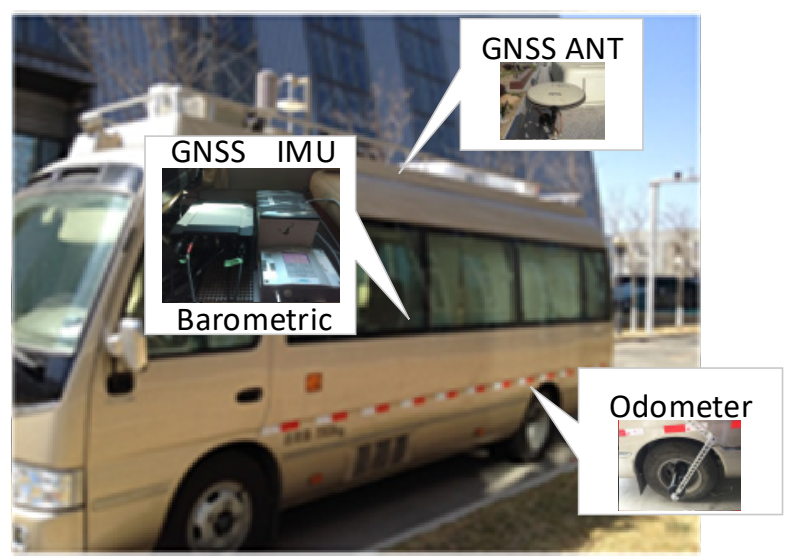

Figure 11. The test platform including GNSS receiver, IMU, barometric altimeter, and odometer.

The test lasts for $3803 \mathrm{~s}$. The trajectory is shown in Figure 12, and the vehicle is driven along the 1-2-3-4 arrow indication. To analyze the performance of the MTF method in different contexts, the test time is divided into: during 0-1999 s and 3300-3803 s, the vehicle is in the open-sky context, and the visible satellites number is almost more than 4 . During 2000-2299 s, the vehicle is in the insufficient satellite context, and two satellites are left, simulating when the GNSS signal is obscured by the building on one side of the road. In Figure 13, the red circles indicate the remaining satellites at 2000 s. During 2300-3299 s, the vehicle is in the satellite outage context, and there is no visible satellite simulating the tunnel. The analysis is conducted on error comparison between the MTF method and traditional GNSS/INS tight-coupled integration.
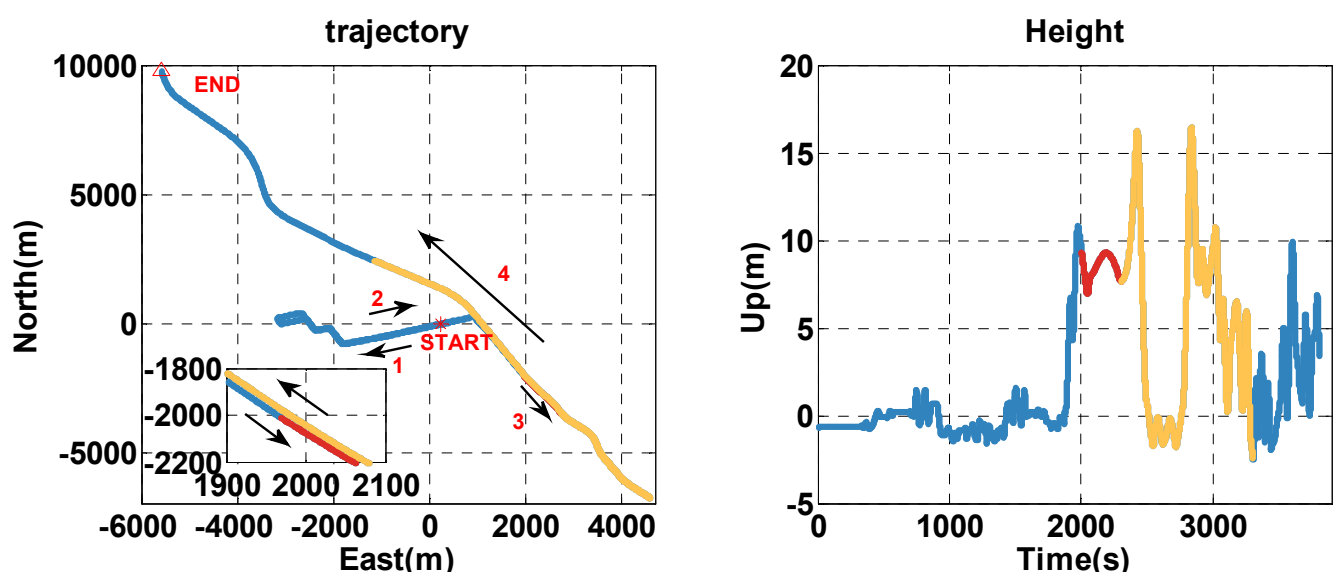

Figure 12. The horizontal trajectory and height of the real-device test. The blue, red, and yellow lines indicate the open-sky, insufficient satellite, and satellite outage parts, respectively.

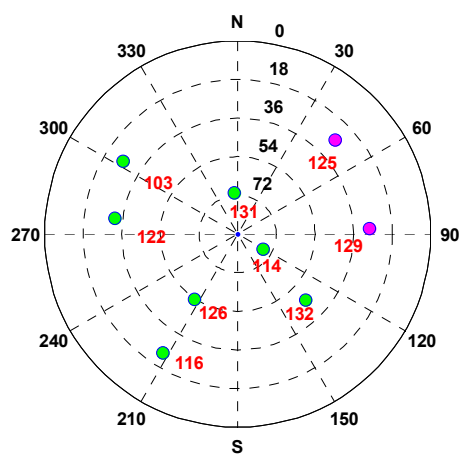

Figure 13. The sky plot of satellites for the epoch of $2000 \mathrm{~s}$, in which green and magenta dots denote the blocked and visible satellites, respectively. 
The noise parameters used in the filter are set as follows. The process noise is set as $0.0012^{\circ} / \sqrt{\mathrm{hr}}$, for the altitude error state and $1250 \mathrm{ug} / \sqrt{\mathrm{Hz}}$ for the velocity error state. The measurement noises are $\sin (E L) / 10 \mathrm{~m}$ and $\sin (E L) / 100 \mathrm{~m} / \mathrm{s}$ for GNSS pseudorange and pseudorange rate, $0.1 \mathrm{~m} / \mathrm{s}$ for odometer velocity, $1 \mathrm{~m}$ for barometric height, and $1 \times 10^{-3} \mathrm{~m}$ for the clock model, respectively.

\subsection{Open-Sky Case}

In the open-sky context (0-1999 s), the position and velocity errors of the MTF method are shown in Figure 14. The position and velocity errors are in the east, north, and up directions, and in the first $300 \mathrm{~s}$, the vehicle is stationary. The position and velocity errors converge at the beginning, and the altitude errors converge after the vehicle moves. To statistically analyze the errors of the MTF method characterized by the 67 th percentile, the orientation error is $0.55^{\circ}$, and the pitch and roll errors are $0.009^{\circ}$ and $0.017^{\circ}$. The horizontal position error is $3.6 \mathrm{~m}$ and the height error is $5.5 \mathrm{~m}$. The horizontal velocity error is $0.08 \mathrm{~m} / \mathrm{s}$ and the vertical velocity error is $0.07 \mathrm{~m} / \mathrm{s}$. The performance of the fusion system is much better than GNSS alone by filtering out the Gaussian noise on the pseudorange and pseudorange rate.
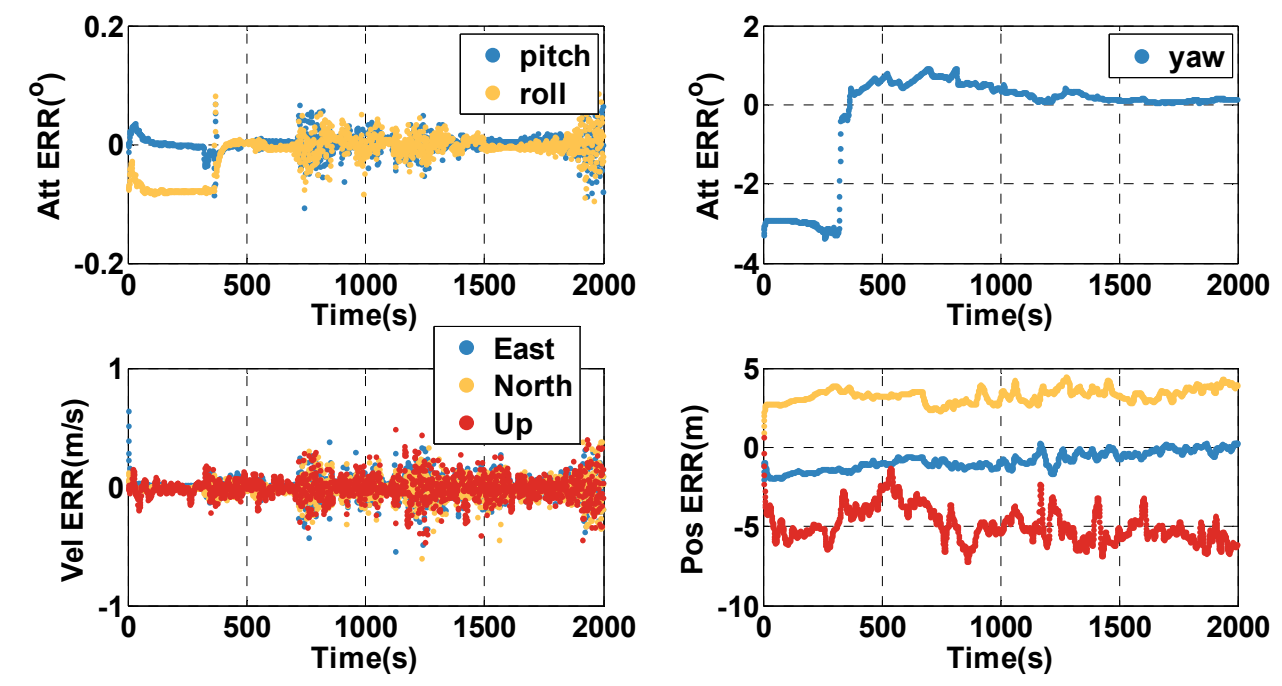

Figure 14. The position, velocity, and attitude errors of the MTF method in the open-sky case.

\subsection{The Insufficient Satellite Case}

The vehicle travel in the insufficient satellite context lasts for 6 min (2000-2299 s), and the vehicle moves toward the southeast in Figure 12. As shown in Figure 13, the remaining visible satellites are presented by magenta dots for the epoch of $2000 \mathrm{~s}$. The position and velocity errors of the MTF method in the east, north, and up directions are shown in Figure 15. The errors of the traditional GNSS/INS tightly coupled integration are shown in Figure 16. The errors of the proposed method do not grow over 6 min in Figure 15, but the errors of GNSS/INS tightly coupled integration increase in Figure 16, especially the position error. In the same time, Figure 15 shows that the error of the MTF method is unstable at the beginning of the travel, and the reason is that the new measurements update causes slight fluctuation in the filter. The error statistics are shown in Table 5, and they are characterized by the 95th percentile. Comparing the MTF method with GNSS/INS, the horizontal position errors of the two methods are approximately $8.73 \mathrm{~m}$ against $31.55 \mathrm{~m}$, the height errors are approximately $6.66 \mathrm{~m}$ against $57.93 \mathrm{~m}$, the horizontal velocity errors are approximately $0.35 \mathrm{~m} / \mathrm{s}$ against $0.32 \mathrm{~m} / \mathrm{s}$, and the vertical velocity errors are approximately $0.32 \mathrm{~m} / \mathrm{s}$ against $0.40 \mathrm{~m} / \mathrm{s}$. Specifically, the statistical horizontal velocity error of the MTF method is a little higher, which is caused by the filter fluctuating at the beginning of the insufficient satellites context when introducing the new measurement update of the barometer geocentric, and the error converges quickly. In sum, the accuracy of the MTF 
method is close to that in the open-sky context, and performs obviously better than GPS/INS tightly coupled integration.
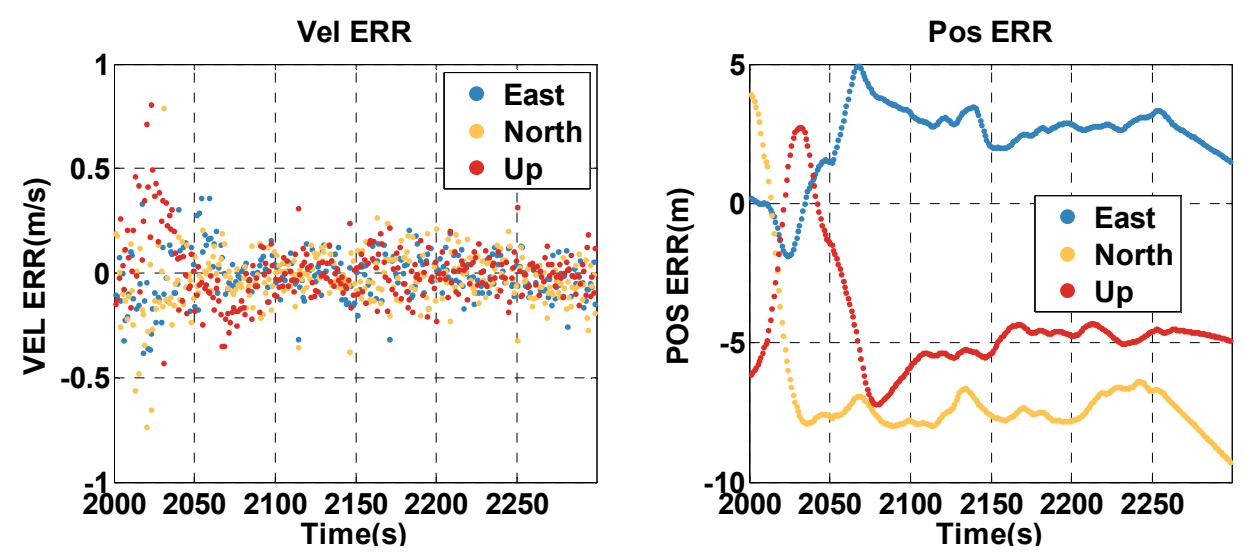

Figure 15. The position and velocity errors of the MTF method in the insufficient satellite case.
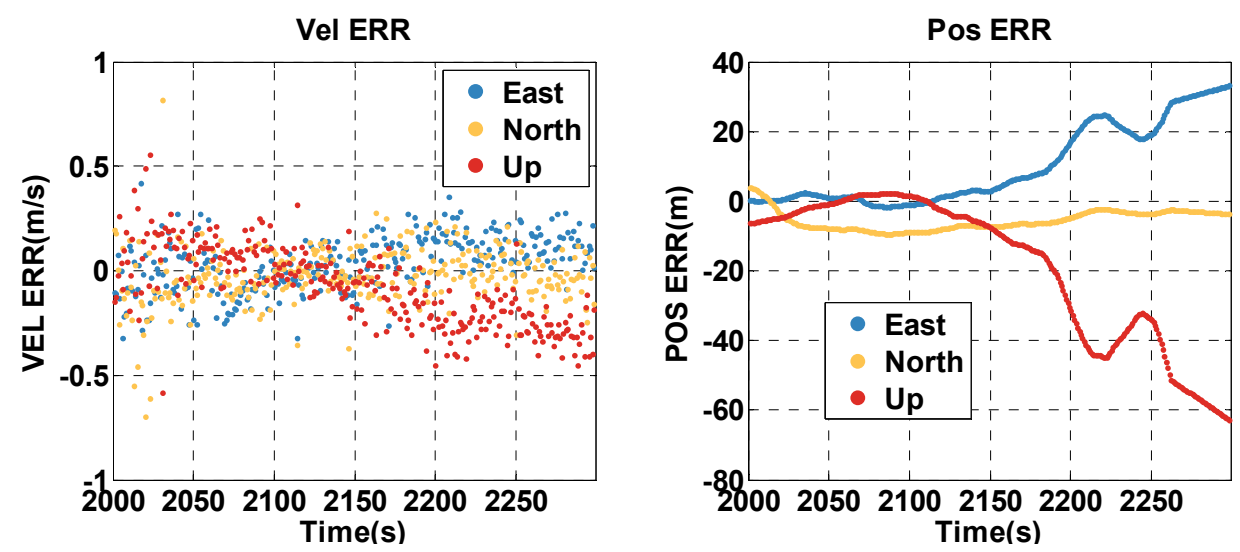

Figure 16. The position and velocity errors of the traditional GNSS/INS tightly coupled integration in the insufficient satellites context in the experimental test.

Table 5. Statistical analysis of the errors of the MTF method and the traditional GNSS/INS tightly coupled integration in the insufficient satellites context in the experimental test.

\begin{tabular}{ccccc}
\hline Method & $\begin{array}{c}\text { Horizontal } \\
\text { Velocity by 95th } \\
\text { Percentile }(\mathbf{m} / \mathbf{s})\end{array}$ & $\begin{array}{c}\text { Vertical Velocity } \\
\text { by 95th Percentile } \\
(\mathbf{m} / \mathbf{s})\end{array}$ & $\begin{array}{c}\text { Horizontal } \\
\text { Position by 95th } \\
\text { Percentile }(\mathbf{m})\end{array}$ & $\begin{array}{c}\text { Height by 95th } \\
\text { Percentile (m) }\end{array}$ \\
\hline MTF & 0.35 & 0.32 & 8.73 & 6.66 \\
GNSS/INS & 0.32 & 0.40 & 31.55 & 57.93 \\
\hline
\end{tabular}

In the case of insufficient satellites, the MTF method predicts the clock error by the clock model. Figure 17 shows the comparison between the clock error estimated by the MTF method and that calculated by GNSS resolution. During 2000-3299 s, there is no GNSS result for insufficient satellites. The clock model works well, as the predicted clock error fits the actual clock error well, before $2000 \mathrm{~s}$ and after $3299 \mathrm{~s}$. 


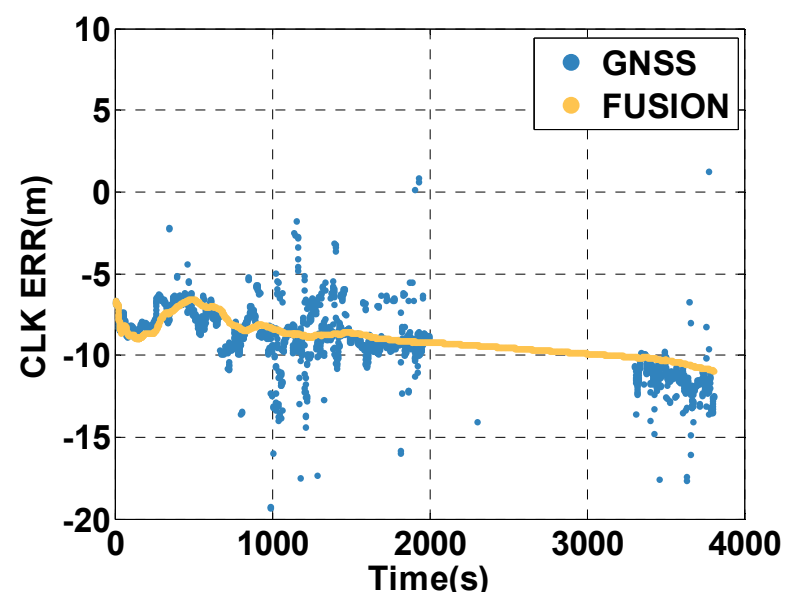

Figure 17. Clock error estimated by the MTF method and by GNSS alone.

\subsection{Satellite Outage Case}

The vehicle travels in the satellite outage context for about $16 \mathrm{~min}$ (2300-3299 s). The position and velocity errors of the MTF method are shown in Figure 18. The position and velocity errors of the traditional GNSS/INS tightly coupled integration are shown in Figure 19. The errors of both methods increase over time, but that of the MTF method is much slower. In addition, the height error does not grow over time. The error statistics are shown in Table 6. The errors are characterized by the 95th percentile. Comparing the MTF method with GNSS/INS, the horizontal position errors of the two methods are approximately $15.52 \mathrm{~m}$ against $184.4 \mathrm{~m}$, the height errors are approximately $5.34 \mathrm{~m}$ against $685.8 \mathrm{~m}$, the horizontal velocity errors are approximately $0.34 \mathrm{~m} / \mathrm{s}$ against $0.78 \mathrm{~m} / \mathrm{s}$, and the vertical velocity errors are approximately $0.27 \mathrm{~m} / \mathrm{s}$ against $1.40 \mathrm{~m} / \mathrm{s}$. The MTF method has obvious advantages in both the horizontal and height directions by the aiding of the odometer and barometric altimeter.
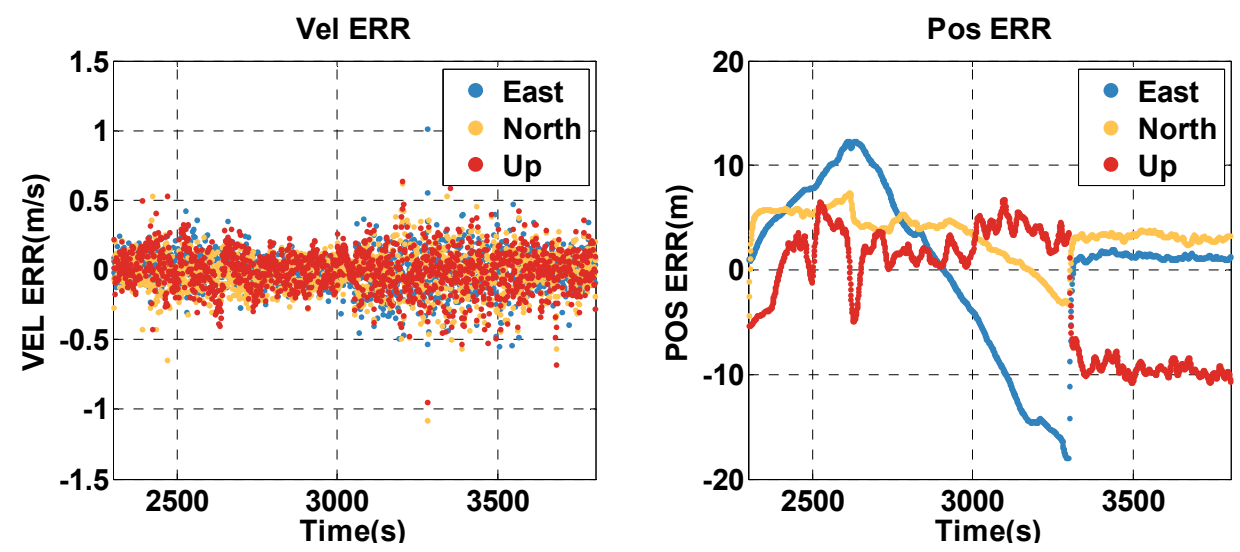

Figure 18. The position and velocity errors of the proposed MTF method in the satellite outage case. 

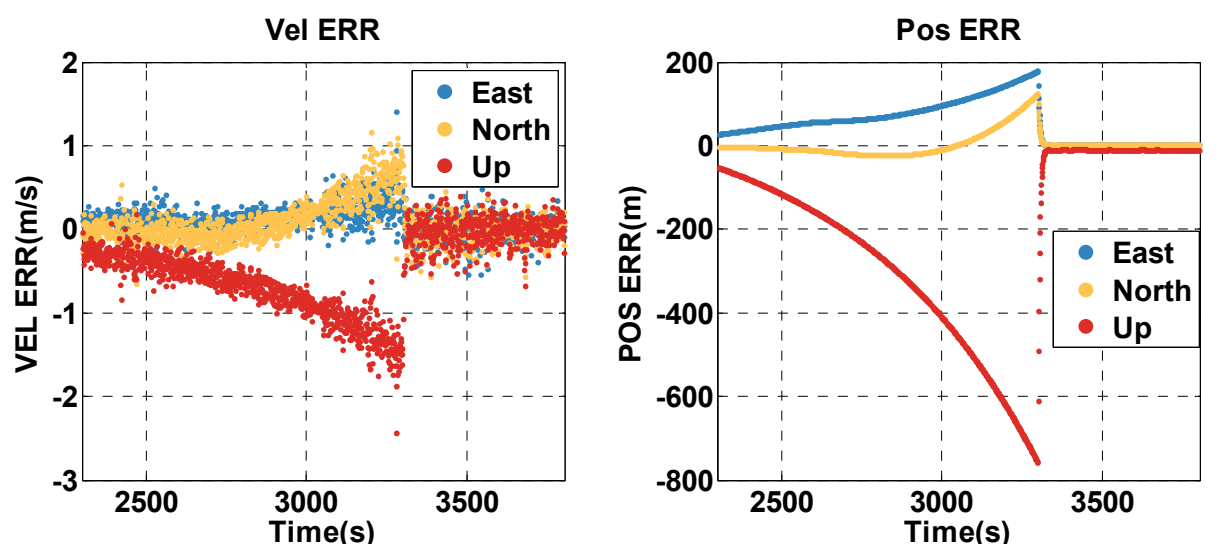

Figure 19. The position and velocity errors of the traditional GNSS/INS tightly coupled integration in the satellite outage case.

Table 6. Statistical analysis of the errors of the MTF method and the traditional GNSS/INS tightly coupled integration in the satellite outage context in the experimental test.

\begin{tabular}{ccccc}
\hline Method & $\begin{array}{c}\text { Horizontal } \\
\text { Velocity by 95th } \\
\text { Percentile }(\mathbf{m} / \mathbf{s})\end{array}$ & $\begin{array}{c}\text { Vertical Velocity } \\
\text { by 95th Percentile } \\
(\mathbf{m} / \mathbf{s})\end{array}$ & $\begin{array}{c}\text { Horizontal } \\
\text { Position by 95th } \\
\text { Percentile }(\mathbf{m})\end{array}$ & $\begin{array}{c}\text { Height by 95th } \\
\text { Percentile (m) }\end{array}$ \\
\hline MTF & 0.34 & 0.27 & 15.52 & 5.34 \\
GNSS/INS & 0.78 & 1.40 & 184.4 & 685.8 \\
\hline
\end{tabular}

\section{Conclusions}

To improve the continuity and reliability of vehicle navigation in complex urban environments, we presented a multi-sensor tight fusion (MTF) method. The fusion method considers GNSS, INS, odometer, barometric altimeter, and clock model sensors, which are easily obtained for vehicles and complement each other. The method designs distinct strategies for the open-sky, insufficient satellites, and satellite outage contexts. In detail, in the open-sky context, the sensor errors can be correctly estimated, which is important as the GNSS signal is impaired. In the insufficient satellites context, the barometric altimeter is treated as a geocentric satellite, combing with the clock model to achieve measurement updates to improve the position accuracy. In the satellite outage context, the odometer and barometric altimeter are used to reduce the horizontal and height divergence of the navigation system. In addition, to reduce the cost of setting up a barometric reference station, the method estimates the error of the barometric altimeter when the GNSS are in good satellite visibility conditions. In sum, the MTF method performs better by adding on other available sensors and a specific fusion strategies design, compared with the traditional GNSS/INS tightly coupled integration.

The simulation test and real-device test were designed to validate the performance of the MTF method. In the simulation test, the sensor errors are preset, and the result shows that the sensor errors estimated by the MTF method match with preset parameters in the open-sky context. In the simulation test and real-device test, the position errors almost do not grow in the insufficient satellites context for $6 \mathrm{~min}$. In the satellite outage context, the position error divergence decreases obviously compared with the traditional GNSS/INS tightly coupled integration. While the validation tests in this paper show that the MTF method performs well, in the future, the method should be modified and optimized to adapt to the actual environment.

Author Contributions: H.Y. and Q.L. conceived the main idea and designed the research. Q.L. implemented the model and algorithms and wrote this paper. D.W. helped analyze the results. N.W. assisted with carrying out the GNSS solution. Z.L. helped carry out GNSS/INS simulations. X.J. assisted with analyzing the data. All authors have read and agreed to the published version of the manuscript.

Funding: This research was funded by the "Collaborative Precision Positioning Project" grant number (No. 2016YFB0501900), XDA13030403, the Strategic Priority Research Program of the Chinese Academy Sciences 
(Grant No: XDA17040202), the BDS Industrialization Project (No. GFZX030302030201-2), the Young Top-Notch Talents Team Program of Beijing Excellent Talents Funding (2017000021223ZK13), and the Youth Innovation Promotion Association and Future Star Program of the Chinese Academy of Sciences.

Conflicts of Interest: The authors declare no conflict of interest.

\section{Appendix A}

$$
\begin{aligned}
& M_{a a}=-\left(\omega_{i e}^{n}+\omega_{e n}^{n}\right) \times \\
& M_{a v}=\left[\begin{array}{ccc}
0 & -1 /(R m+h) & 0 \\
1 /(R n+h) & 0 & 0 \\
1 /(R n+h) \cdot \tan (\text { Lan }) & 0 & 0
\end{array}\right] \\
& M_{a p}=\left[\begin{array}{ccc}
0 & 0 & v(2) /(R m+h)^{2} \\
-\omega_{i e}^{n}(3) & 0 & -v(1) /(R n+h)^{2} \\
\omega_{i e}^{n}(2)+v(1) /(R n+h) \cdot \sec ^{2}(\text { Lan }) & 0 & -v(1) /(R n+h)^{2} * \tan (\text { Lan })
\end{array}\right] \\
& M_{v a}=\left(\mathrm{C}_{b}^{n} \cdot f_{b}\right) \times \\
& M_{v v}=(v) \times M_{a v}-\left(2 \omega_{i e}^{n}+\omega_{e n}^{n}\right) \times \\
& M_{v p}=v \times\left[\begin{array}{ccc}
0 & 0 & v(2) /(R m+h)^{2} \\
-2 \omega_{i e}^{n}(3) & 0 & -v(1) /(R n+h)^{2} \\
2 \omega_{i e}^{n}(2)+v(1) /(R n+h) \cdot \sec ^{2}(\text { Lan }) & 0 & -v(1) /(R n+h)^{2} \cdot \tan (\text { Lan })
\end{array}\right] \\
& M_{p v}=\left[\begin{array}{ccc}
0 & 1 /(R m+h) & 0 \\
1 /(R n+h) & 0 & 0 \\
0 & 0 & 1
\end{array}\right] \\
& M_{v p}=\left[\begin{array}{clc}
0 & 0 & -v(2) /(R m+h)^{2} \\
v(1) /(R n+h) \cdot \tan (\text { Lan }) \sec (\text { Lan }) & 0 & -v(1) /(R n+h)^{2} \cdot \sec (\text { Lan }) \\
0 & 0 & 0
\end{array}\right] \\
& \omega_{i e}^{n}=\left[\begin{array}{c}
0 \\
\omega_{i e} \cos (\text { Lan }) \\
\omega_{i e} \sin (\text { Lan })
\end{array}\right] \\
& \omega_{e n}^{n}=\left[\begin{array}{c}
-\frac{v(2)}{R m+h} \\
\frac{v(1)}{R n+h} \\
\frac{v(1)}{R n+h} \tan (\text { Lan })
\end{array}\right]
\end{aligned}
$$

where $R m$ is the radius of curvature along the meridian circle, and $R n$ is the radius of curvature along the meridional circle. Lan is the longitude, $h$ is the height, and $v$ is the velocity in the local frame. $\omega_{i e}$ is the earth's rotation angular velocity, and $f_{b}$ is the specific force from the accelerometer sensor.

\section{References}

1. Fernández, E.; David, C.; Parés, M. CSAC Characterization and Its Impact on GNSS Clock Augmentation Performance. Sensors 2017, 17, 370. [CrossRef] [PubMed]

2. Ding, W.; Wang, J.; Rizos, C.; Kinlyside, D. Improving Adaptive Kalman Estimation in GPS/INS Integration. J. Navig. 2007, 60, 517. [CrossRef]

3. Yao, Y.; Xu, X.; Zhu, C.; Chan, C.Y. A hybrid fusion algorithm for GPS/INS integration during GPS outage. Measurement 2017, 103, 42-51. [CrossRef]

4. Li, Z.; Gao, J.; Wang, J.; Yao, Y. PPP/INS tightly coupled navigation using adaptive federated filter. GPS Solut. 2017, 21, 137-148. [CrossRef] 
5. Gianluca, F.; Marco, P.; Gianluca, M. Loose and Tight GNSS/INS Integrations: Comparison of Performance Assessed in Real Urban Scenarios. Sensors 2017, 17, 255.

6. Godha, S.; Cannon, E. GPS/MEMS INS integrated system for navigation in urban areas. GPS Solut. 2007, 11, 193-203. [CrossRef]

7. Chiang, K.-W.; Duong, T.T.; Liao, J.K. The Performance Analysis of a Real-Time Integrated INS/GPS Vehicle Navigation System with Abnormal GPS Measurement Elimination. Sensors 2013, 13, 10599-10622. [CrossRef] [PubMed]

8. Yi, D.; Dingjie, W.; Liang, Z.; Qingsong, L.; Jie, W. Tightly Coupled GNSS/INS Integration with Robust Sequential Kalman Filter for Accurate Vehicular Navigation. Sensors 2020, 20, 561.

9. Paziewski, J. Recent advances and perspectives for positioning and applications with smartphone GNSS observations. Meas. Sci. Technol. 2020, 1-14. [CrossRef]

10. Walter, O.; Schmalenstroeer, J.; Engler, A.; Haeb-Umbach, R. Smartphone-Based Sensor Fusion for Improved Vehicular Navigation. In 2013 10th Workshop on Positioning, Navigation and Communication (WPNC); IEEE: Dresden, Germany, 2013.

11. Georgy, J.; Noureldin, A.; Korenberg, M.J. Bayoumi, M.M. Low-Cost Three-Dimensional Navigation Solution for RISS/GPS Integration Using Mixture Particle Filter. IEEE Trans. Veh. Tech. 2010, 59, 599-615. [CrossRef]

12. Falco, G.; Nicola, M.; Pini, M. Positioning Based on Tightly Coupled Multiple Sensors: A Practical Implementation and Experimental Assessment. IEEE Access 2018, 6, 13101-13116. [CrossRef]

13. Fei, L.; Houzeng, H.; Xin, C.; Binghao, L. Performance of Tightly Coupled Integration of GPS/BDS/MEMS-INS/Odometer for Real-Time High-Precision Vehicle Positioning in Urban Degraded and Denied Environment. J. Sens. 2020, 2020, 1-15.

14. Georgy, J.; Karamat, T.; Iqbal, U. Noureldin, A. Enhanced MEMS-IMU/odometer/GPS integration using mixture particle filter. GPS Solut. 2011, 15, 239-252. [CrossRef]

15. Li, Z.; Wang, J.; Li, B.; Gao, J.; Tan, X. GPS/INS/Odometer Integrated System Using Fuzzy Neural Network for Land Vehicle Navigation Applications. J. Navig. 2014, 67, 967-983. [CrossRef]

16. Parviainen, J.; Kantola, J.; Collin, J. Differential barometry in personal navigation. In 2008 IEEE/ION Position, Location E Navigation Symposium; IEEE: Piscataway, NJ, USA, 2008; pp. 148-152.

17. Yen, S.W.; van Graas, F.; de Haag, M.U. Positioning with two satellites and known receiver clock, barometric pressure and radar elevation. GPS Solut. 2015, 20, 885-899. [CrossRef]

18. Chiang, K.W.; Chang, H.W.; Li, Y.H.; Tsai, G.J.; Tseng, C.L.; Tien, Y.C.; Hsu, P.C. Assessment for INS/GNSS/Odometer/Barometer Integration in Loosely-Coupled and Tightly-Coupled Scheme in a GNSS-Degraded Environment. IEEE Sens. J. 2020, 20, 3057-3069. [CrossRef]

19. Mcburney, P.W.; Brown, R.G. Receiver clock stability-An important aid in the GPS integrity problem. In Proceedings of the Institute of Navigation, National Technical Meeting, Santa Barbara, CA, USA, 26-29 January 1988; pp. 237-244.

20. Wu, P.; Jing, S.; Liu, W.; Wang, F. A Quick Location Method for High Dynamic GNSS Receiver Based on Time Assistance. Int. J. Comput. Commun. Control 2013, 8, 754. [CrossRef]

21. Krawinkel, T.; Schon, S. Benefits of receiver clock modeling in code-based GNSS navigation. GPS Solut. 2016, 20, 687-701. [CrossRef]

22. Li, B.; Zhang, H.; Wang, W.; Li, X. BDS in Challenge Environment Using CSAC. In Proceedings of the 2019 Joint Conference of the IEEE International Frequency Control Symposium and European Frequency and Time Forum (EFTF/IFC), Orlando, FL, USA, 14-18 April 2019. [CrossRef]

23. Zhou, P.; Zheng, Y.; Li, Z.; Li, M.; Shen, G. IODetector: A Generic Service for Indoor/Outdoor Detection. In Proceedings of the 10th ACM Conference on Embedded Network Sensor Systems, Toronto, ON, Canada, 6-9 November 2012; Volume 11, pp. 361-362.

24. Groves, P.D.; Wang, L.; Walter, D.; Martin, H.; Voutsis, K. Toward a Unified PNT, Part 1: Complexity and context: Key challenges of multisensor positioning. GPS World 2014, 25, 18-49.

25. Saeedi, S.; Moussa, A.; El-Sheimy, N. Context-Aware Personal Navigation Using Embedded Sensor Fusion in Smartphones. Sensors 2014, 14, 5742-5767. [CrossRef] [PubMed]

26. Kaplan, E.; Hegarty, C. Understanding GPS: Principles and Applications, 3rd ed.; Artech House: Fitchburg, MA, USA, 2017.

27. Titterton, D.; Weston, J.L. Strapdown Inertial Navigation Technology, 2nd ed.; IET: London, UK, 2004. 
28. Liu, K.; Wang, Y.; Wang, J. Differential Barometric Altimetry Assists Floor Identification in WLAN Location Fingerprinting Study. In Principle and Application Progress in Location-Based Services; Springer International Publishing: Berlin/Heidelberg, Germany, 2014; pp. 21-29.

29. National Oceanic and Atmospheric Administration. U.S. Standard Atmosphere; U.S. Government Printing Office: Washington, DC, USA, 1976.

(C) 2020 by the authors. Licensee MDPI, Basel, Switzerland. This article is an open access article distributed under the terms and conditions of the Creative Commons Attribution (CC BY) license (http://creativecommons.org/licenses/by/4.0/). 